\title{
THERMODYNAMICS BASED ARTIFICIAL NEURAL NETWORKS BASED HOMOGENIZATION OF COMPOSITE MATERIALS
}

\author{
$\underline{\text { S. Müller }}^{1^{*}}$ \\ ${ }^{1}$ ESI Group, Germany \\ *Sebastian.mueller@esi-group.com
}

The macroscopic performance of modern composite materials is determined by the material behaviour of the individual constituents as well as the material architecture. The discrepancy in length scale between the latter and the composite part dimensions prevent a direct modelling of the material structure in an industrial relevant part simulation. Multiscale based analysis in combination with homogenization techniques are commonly used to determine the effective material properties. The subsequent macro scale simulation however requires the availability of suitable constitutive models. The sheer variability in the possible constituents as well as in the material micro- and meso-structure hinder the formulation of universal material models.

Machine learning based material modelling has therefore gained increasing attention [1]. Approaches like neural networks allow for a fast implementation of complex relations and have been proven to give good accuracy in predicting the effective mechanical response of complex material micro-structures [2] and inelastic material behaviour [3].

The generic implementation of the neural network evaluation functions in a commercial Finite Element code, such as ESI Virtual Performance Solution (VPS) [4] allows for the direct application of the method to industrial relevant problems. In the present contribution a feed forward neural network is used to describe the effective material behaviour of composites. A principle sketch of the network architecture is given in Fig. 1 (a). To investigate the applicability of the approach for a variety of composite materials, continuous and discontinuous fibre reinforced polymers are analysed (e.g. Fig. 1 (b)).

(a)

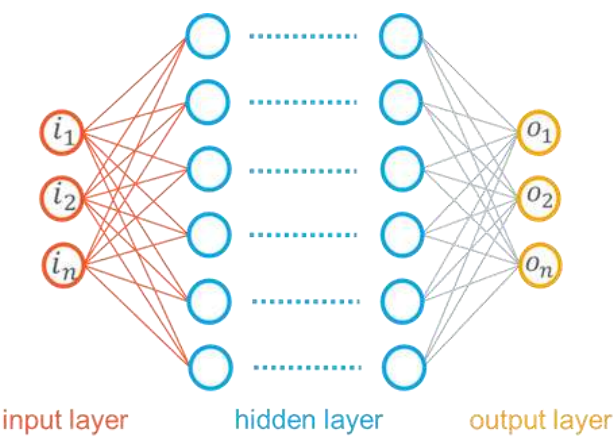

(b)

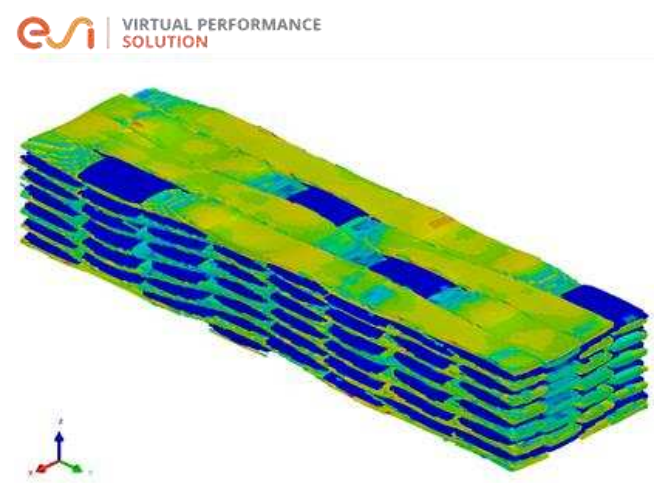

Figure 1. (a) Feed forward neural network architecture, (b) 3D textile reinforced composite - unit cell of the mesoscopic material structure

\section{References}

[1] Liu, X., Tian, S., Tao, F., Du, H., \& Yu, W. (2020). "How machine learning can help the design and analysis of composite materials and structures". ArXiv: Materials Science.

[2] Liu, Z., Wei, H., Huang, T., \& Wu, C. (2020). "Intelligent multiscale simulation based on process-guided composite database". ArXiv, abs/2003.09491.

[3] Masi, F., I. Stefanou, P. Vannucci and V. Maffi-Berthier. (2020) "Thermodynamicsbased Artificial Neural Networks for constitutive modeling”. ArXiv abs/2005.12183.

[4] ESI Group, Virtual Performance Solution, https://www.esi-group.com. 